\title{
Spatial walking solitons in quadratic nonlinear crystals
}

\author{
Lluis Torner, Dumitru Mihalache,* Dumitru Mazilu,* and Maria C. Santos \\ Laboratory of Photonics, Department of Signal Theory and Communications, Universitat Politecnica de Catalunya, \\ Gran Capitan UPC-D3, Barcelona ES 08034, Spain
}

Nail N. Akhmediev

Optical Sciences Center, Institute of Advanced Studies, The Australian National University, Canberra, ACT 0200, Australia

Received June 10, 1997; revised manuscript received January 22, 1998

\begin{abstract}
We investigate the properties of the families of spatial walking solitons propagating in planar waveguides made of quadratic nonlinear media under conditions for second-harmonic generation in the presence of Poynting vector walk-off. We study in detail the shape and various features of the solitons, their stability on propagation, and their excitation. (C) 1998 Optical Society of America [S0740-3224(98)00904-7]
\end{abstract}

OCIS code: 190.0190 .

\section{INTRODUCTION}

Optical solitons in quadratic nonlinear media ${ }^{1}$ are a subject of intense investigation. The interest in the issue is driven by the rich dynamic features that are continually uncovered and by their potential applications to the manipulation of light signals in different situations. ${ }^{2-13}$ Those applications include single-pass geometries and optical cavities containing quadratic nonlinear crystals, in settings for spatial, temporal, and spatiotemporal trapping of light beams and pulses. Here we study the spatial case. Solitons (or, more properly, solitary waves) exist in a wide variety of material and input light conditions in both bulk crystals and planar waveguides, and they were observed experimentally by Torruellas et al. ${ }^{14}$ and by Schiek and co-workers. ${ }^{15}$

By their very nature, solitons in quadratic nonlinear media are made from the mutual trapping of several waves. Here we consider the formation of spatial solitons under conditions for second-harmonic generation; therefore the solitons exist because of the mutual trapping of the fundamental and the second-harmonic beams. In general, except under experimentally suitable conditions, in the low-power regime the beams propagate along different directions because of the Poynting vector walkoff that is present in anisotropic media. This fact has important experimental implications when it comes to the choice of suitable materials, input-light wavelength, and general conditions suitable to the formation of solitons.

However, when a soliton is formed the interacting waves are mutually trapped, and in the presence of Poynting vector walk-off the beams drag each other and propagate stuck, or locked together. Such beam locking opens the possibility to specific applications of the solitons ${ }^{16-19}$ and also poses new challenges to the understanding of soliton formation. This is so because the walking solitons that exist in the presence of walk-off exhibit new fea- tures in comparison with the nonwalking solitons. Investigation of these new features is important for their potential applications but also from a fundamental viewpoint because the approach and outcome have implications for the existence of walking solitons in other analogous but different physical settings.

Our goal in this paper is to report the results of our comprehensive investigations of the properties of the existing families of spatial walking solitons. Families of walking solitons exist in $(1+1)$ geometries and $(2$ +1 ) geometries. ${ }^{20-22}$ Here we restrict ourselves to the one-dimensional case, but most general features hold also in the bulk case. We study in detail the amplitude and wave-front shapes of the solitons, analyze the stability on propagation of the members of the families of stationary solutions, and discuss the salient points of their excitation with different input beams.

The remainder of this paper is organized as follows. In Section 2 we present the governing evolution equations and discuss briefly the meaning of the various parameters involved. In Section 3 we obtain the equations that give the families of walking solitons in the frame of reference moving with the soliton peak, and we discuss the general properties of the solitons that can be obtained from the conserved quantities of the wave evolution. Sections 4 and 5 are devoted to the detailed properties of the families of solitons, including their cutoff conditions, similarity rules, and shape features, with special attention to their dependence on the soliton energy and velocity. In Section 6 we study the stability on propagation of the solitons, and in Section 7 we discuss their excitation. Finally, in Section 8 we summarize our main conclusions.

\section{GOVERNING EQUATIONS}

We consider cw light beams traveling in a medium with a large quadratic nonlinearity under conditions of second- 
harmonic generation. Here we focus on spatial solitons in $(1+1)$ geometries, i.e., solitons in planar waveguides and in type I phase-matching settings, but the approach reported also holds for $(2+1)$ walking solitons in bulk crystals and also for type II phase matching. Naturally, the families of walking solitons are richer in the case of type II geometries because of the additional degree of freedom that this phase-matching setting offers, but type I geometries are simpler and capture the specific features of the walking solitons. Hence we focus here on them. In the slowly varying envelope approximation the beam evolution is described by the reduced normalized equations (see, e.g., Ref. 6)

$$
\begin{array}{r}
i \frac{\partial a_{1}}{\partial \xi}-\frac{r}{2} \frac{\partial^{2} a_{1}}{\partial s^{2}}+a_{1} * a_{2} \exp (-i \beta \xi)=0, \\
i \frac{\partial a_{2}}{\partial \xi}-\frac{\alpha}{2} \frac{\partial^{2} a_{2}}{\partial s^{2}}-i \delta \frac{\partial a_{2}}{\partial s}+a_{1}{ }^{2} \exp (i \beta \xi)=0,
\end{array}
$$

where $a_{1}$ and $a_{2}$ are the normalized amplitudes of the fundamental and the second-harmonic waves, respectively, $r=-1$ for spatial solitons, and $\alpha=-k_{1} / k_{2}$. Here $k_{1,2}$ are the linear wave numbers at both frequencies. In all cases $\alpha \simeq-0.5$, so we set $\alpha=-0.5$. The parameter $\beta$ is given by $\beta=k_{1} \eta^{2} \Delta k$, where $\Delta k=2 k_{1}$ $-k_{2}$ is the wave-vector mismatch and $\eta$ is a characteristic beam width. In Eqs. (1) the transverse coordinates are given in units of $\eta$, and the scaled propagation coordinate is $\xi=z / k_{1} \eta^{2}$. Therefore, for a beam width of some $\eta \sim 15 \mu \mathrm{m}$, which yields a diffraction length $l_{d 1}$ $=k_{1} \eta^{2} / 2 \sim 1 \mathrm{~mm}, \xi$ in the range $0-20$ corresponds to a few centimeters.

The parameter $\delta$ is central to our analysis because it accounts for the Poynting vector walk-off that occurs in birefringent media when propagation is not along the crystal optical axes. Nonlinear quadratic optical materials are noncentrosymmetric, anisotropic crystals; therefore walk-off is always present in the experiments when birefringence-tuning phase-matching techniques are used. Most crystals with large $\chi^{(2)}$ nonlinear coefficients are also highly birefringent, as is the case for many organics, and for that case large values of $\delta$ will result for angle-tuned configurations. Under such conditions, Eqs. (1) may not be valid. Walk-off is absent in noncritical and quasi-phase-matching geometries ${ }^{23}$ because in those cases propagation does occur along the crystalline optical axes.

Equations (1) also hold for pulsed light. Then diffraction is replaced by dispersion, Poynting vector walk-off is replaced by temporal walk-off owing to group-velocity mismatch, and $r$ and $\alpha$ are given by the group-velocity dispersions at the fundamental and at the secondharmonic frequencies, respectively.

\section{WALKING SOLITONS}

We study stationary solutions of Eqs. (1) describing mutually trapped beams walking off the $\xi=0$ axis. Those solutions have the form

$$
a_{\nu}(\xi, s)=U_{\nu}(\eta) \exp \left[i \phi_{\nu}(\xi, \eta)\right], \quad \nu=1,2,
$$

where $U$ and $\phi$ are real functions, $\eta=s-v \xi$ is the transverse coordinate moving with the soliton peak, and $\phi_{\nu}(\xi, s)=\kappa_{\nu} \xi+f_{\nu}(\eta)$. Here $v$ is the soliton velocity, $\kappa_{\nu}$ are the nonlinear wave-number shifts produced by the wave interaction, and $f_{\nu}(\eta)$ stand for the transverse wave fronts of the solitons. To avoid all energy exchange between the waves one needs $\kappa_{2}=2 \kappa_{1}+\beta$. Also, the wave fronts have to verify that $f_{2}(\eta)=2 f_{1}(\eta)$ everywhere, or alternatively $U_{\nu}(\eta)$ and $f_{\nu}(\eta)$ ought to be symmetric and antisymmetric functions, respectively, of the transverse coordinate $\eta$. It turns out that only the traveling-wave soliton solutions that occur in the absence of walk-off fulfill the former condition, whereas with this exception all the walking solitons fulfill the latter. This has interesting consequences, which are discussed below.

Substitution of Eq. (2) into Eqs. (1) yields the system of coupled nonlinear ordinary differential equations fulfilled by the functions $U_{\nu}(\eta)$ and $f_{\nu}(\eta)$. One has

$$
\begin{gathered}
1 /{ }_{2} \ddot{U}_{1}-\left(\kappa_{1}-v \dot{f}_{1}+1 / 2 \dot{f}_{1}^{2}\right) U_{1}+U_{1} U_{2} \cos \left(f_{2}-2 f_{1}\right) \\
=0, \\
1 / 2 \ddot{f}_{1} U_{1}+\left(\dot{f}_{1}-v\right) \dot{U}_{1}+U_{1} U_{2} \sin \left(f_{2}-2 f_{1}\right)=0, \\
1 / 2 \alpha \ddot{U}_{2}+\left[2 \kappa_{1}+\beta-(v+\delta) \dot{f}_{2}-1 / 2 \alpha \dot{f}_{2}^{2}\right] U_{2} \\
-U_{1}^{2} \cos \left(f_{2}-2 f_{1}\right)=0, \\
1 / 2 \alpha \ddot{f}_{2} U_{2}+\left(\alpha \dot{f}_{2}+v+\delta\right) \dot{U}_{2}+U_{1}^{2} \sin \left(f_{2}-2 f_{1}\right)
\end{gathered}
$$

where the overdots indicate the derivative with respect to $\eta$. Recall that $\alpha, \beta$, and $\delta$ are given by linear wave parameters, whereas the nonlinear wave-number shift $\kappa_{1}$ and the velocity $v$ parameterize the families of walking solitons.

The system of Eqs. (3)-(6) has a trivial traveling-wave solution with the form of Eq. (2) with the phase front $f_{\nu}(\eta)=\omega_{\nu} \eta$. However, substitution into the equations gives $\omega_{1}=v, \omega_{2}=-(\delta+v) / \alpha$, and $v=-\delta /(2 \alpha+1)$. Because in all experimental situations one has $\alpha$ $\simeq-0.5$, this expression gives a value of $v$ orders of magnitude larger than the actual soliton velocity. Therefore the corresponding solutions do not have physical relevance unless $\alpha=-0.5$ and there is no walk-off so that $\delta=0$. In general, the physically relevant families of walking solitons do not have the above simple wave-front tilts, but rather they have nontrivial, curved wave fronts. Their analytical form is not known at present, so the families of walking solitons ought to be calculated numerically.

Important information about the families of walking solitons can be obtained from the conserved quantities of the wave evolution, as follows. ${ }^{24}$ Governing equations (1) constitute an infinite-dimensional Hamiltonian dynamical system, with the conserved Hamiltonian

$$
\begin{aligned}
H= & -\frac{1}{2} \int\left[r\left|\frac{\partial A_{1}}{\partial s}\right|^{2}+\frac{\alpha}{2}\left|\frac{\partial A_{2}}{\partial s}\right|^{2}-\beta\left|A_{2}\right|^{2}\right. \\
& +i \frac{\delta}{2}\left(A_{2} \frac{\partial A_{2}^{*}}{\partial s}-A_{2} * \frac{\partial A_{2}}{\partial s}\right) \\
& \left.+\left(A_{1}^{* 2} A_{2}+A_{1}^{2} A_{2} *\right)\right] \mathrm{d} s,
\end{aligned}
$$


where we have defined $A_{1}=a_{1}$ and $A_{2}=a_{2} \exp (-i \beta \xi)$. We shall also make use of two additional conserved quantities: the total intensity or energy flow given by the Manley-Rowe relation

$$
I=I_{1}+I_{2}=\int\left[\left|A_{1}\right|^{2}+\left|A_{2}\right|^{2}\right] \mathrm{d} s
$$

and the total transverse beam momentum

$$
\begin{aligned}
J= & J_{1}+J_{2}=\frac{1}{4 i} \int\left[2\left(A_{1} * \frac{\partial A_{1}}{\partial s}-A_{1} \frac{\partial A_{1} *}{\partial s}\right)\right. \\
& \left.+\left(A_{2} * \frac{\partial A_{2}}{\partial s}-A_{2} \frac{\partial A_{2} *}{\partial s}\right)\right] \mathrm{d} s .
\end{aligned}
$$

One finds that the stationary walking solitons with the form given by Eq. (2) occur at the extrema of the Hamiltonian for a given energy flow and a given transverse momentum; i.e., they occur at

$$
\delta_{F}\left(H+\kappa_{1} I-v J\right)_{\mathrm{sta}}=0,
$$

where $\delta_{F}$ stands for the Fréchet or variational derivative. This is an important result that has important implications for soliton stability, as we discuss in Section 6. For now, using either the Derrick-Hobart theorem or directly manipulating the governing equations, we use this result to find that actually the stationary walking soliton solutions are realized at the value of the Hamiltonian

$$
H=-\frac{3}{5} \kappa_{1} I+\frac{1}{5} \beta I_{2}+\frac{4}{5} v J-\frac{1}{5} \delta J_{2} .
$$

When $\delta=0$ the last two terms on the right-hand side of Eq. (11) vanish for zero-velocity solutions. However, in the presence of walk-off only the third term vanishes for zero-velocity solitons, whereas the last term contributes to the Hamiltonian. This is an indication that the transverse momentum of the walking solitons is not simply proportional to their velocity, unlike for walking solitons of Galilean invariant evolution equations. Substitution of Eq. (2) into Eq. (9) yields

$$
J=1 / 2 \int\left(2 U_{1}^{2} \dot{f}_{1}+U_{2}^{2} \dot{f}_{2}\right) \mathrm{d} s .
$$

In general $\dot{f}_{1,2}$ are not simply proportional to the soliton velocity; therefore neither is $J$. We can elucidate the actual relation between the velocity and the momentum for the stationary walking solitons by examining the evolution of the energy centroid of the bound state constituted by the fundamental and the second-harmonic beams propagating stuck together. We find that ${ }^{16}$

$$
J=I v+\delta I_{2}+(2 \alpha+1) J_{2} .
$$

In contrast to the above results, the Galilean-boosted traveling-wave solutions that occur when $\delta=0$ with $\alpha$ $=-0.5$ have a flat wave front and a wave-front tilt given by the soliton velocity. In such conditions we find the Galilean particlelike results

$$
\begin{aligned}
& J=I v, \\
& H=H_{v=0}+1 / 2 I v^{2},
\end{aligned}
$$

where $H_{v=0}$ is the Hamiltonian of the zero-velocity solitons and the term $I v^{2} / 2$ represents the kinetic part of the
Hamiltonian of the solitons walking with velocity $v$. However, Eqs. (11) and (13) show that such is not the case for the solitons that we are investigating because of their wave-front curvatures.

Eventually, we notice that Eq. (10) provides the starting point of a variational method to find approximate analytical expressions of the families of walking solitons. The procedure requires setting judicious trial functions for the soliton amplitude and wave front, calculating the corresponding $I, J$, and $H$, and then optimizing the parameters of the trial function to minimize $H$. However, our goal here is to examine the exact properties of the families of solitons; therefore we consider the exact numerical solution of the governing equations.

\section{CUTOFF AND SIMILARITY RULES}

Stationary, walking soliton solutions of Eqs. (3)-(6) exist for nonlinear wave-number shifts $\kappa_{1}$ and transverse velocities $v$ such that the soliton is not in resonance with linear dispersive waves. Otherwise, the coupling between the waves would lead to energy leakage emitted from the soliton. ${ }^{25}$ The resonance condition can be calculated by matching the behavior at $\eta \rightarrow \pm \infty$ of the longitudinal components of the nonlinear wave numbers of the two waves forming the soliton, i.e., $q_{\nu, \mathrm{nl}}=\kappa_{\nu}-v \dot{f}_{\nu}(\eta)$, with the corresponding wave numbers of linear waves $q_{\nu \text {,lin. }}$. The latter are given by the linearized version of Eqs. (3)-(6), but such is not necessarily so for $q_{\nu, \mathrm{nl}}$.

To elucidate the behavior of $q_{\nu, \mathrm{nl}}$ at the soliton tails, we proceed as follows. For the fundamental beam, setting $U_{1} \sim \exp \left(-\Gamma_{1} \eta\right)$ and linearizing Eqs. (3) and (4) give $\Gamma_{1}^{2}=2 \kappa_{1}-v^{2}$ and

$$
\dot{f}_{1}(\eta \rightarrow \infty)=v \text {. }
$$

In general, the tails of the second-harmonic beam are more complicated because the nonlinear terms in Eqs. (5) and (6) may decay at $\eta \rightarrow \pm \infty$ with the same rate as the linear terms. When such is not the case, one has $U_{2}$ $\sim \exp \left(-\Gamma_{2} \eta\right)$, with $\Gamma_{2}<2 \Gamma_{1}$. Then, Eqs. (5) and (6) yield

$$
\begin{gathered}
\Gamma_{2}^{2}=-\frac{2}{\alpha}\left[2 \kappa_{1}+\beta+\frac{(v+\delta)^{2}}{2 \alpha}\right], \\
\dot{f}_{2}(\eta \rightarrow \infty)=-\frac{1}{\alpha}(\delta+v) .
\end{gathered}
$$

However, for the condition $\Gamma_{2}<2 \Gamma_{1}$ to be fulfilled by Eq (17) it is necessary that

$$
\frac{1}{\alpha}\left[(2 \alpha+1)\left(2 \kappa_{1}-v^{2}\right)+v^{2}+\beta+\frac{(v+\delta)^{2}}{2 \alpha}\right]>0 .
$$

In the case $\alpha=-0.5$ that we always consider in the numerics here, inequality (19) leads to

$$
\delta(2 v+\delta)>\beta .
$$

Then resonance of the second-harmonic beam with linear waves occurs below:

$$
\kappa_{1, \text { cut }}^{(2)}=1 / 2\left[(\delta+v)^{2}-\beta\right] .
$$


In general, including the case in which $\Gamma_{2}=2 \Gamma_{1}$ and those in which the second-harmonic tails are not single exponentials, cutoff of the fundamental wave occurs at

$$
\kappa_{1, \text { cut }}^{(1)}=1 / 2 v^{2} \text {. }
$$

Therefore, for given values of the various involved parameters, stationary walking solitons exist for nonlinear wave-number shifts above these cutoff values. Outside the region in parameter space where condition (19) is verified, the tails of the wave front of the second-harmonic beam of the walking solitons are more complicated. In particular, in general $f_{2}$ can exhibit oscillating tails that are due to the last term of Eqs. (5) and (6).

We can extract useful information about the families of walking solitons by examining the scaling properties of Eqs. (3)-(6). We readily find that such equations are invariant under the transformations

$$
\begin{array}{rlrl}
\kappa_{1} \rightarrow \mu \kappa_{1}, \quad v & \rightarrow \sqrt{\mu} v, \quad \beta \rightarrow \mu \beta, \quad \delta \rightarrow \sqrt{\mu} \delta, \\
U_{1} & \rightarrow \mu U_{1}, & U_{2} & \rightarrow \mu U_{2}, \\
s & \rightarrow s / \sqrt{\mu}, \quad I \rightarrow \mu^{3 / 2} I,
\end{array}
$$

with $\mu \neq 0$ being an arbitrary parameter. Thus we can transform the soliton solutions into each other by following such rules.

This self-similarity has important consequences. For example, it shows that for given values of the soliton velocity $v$ and the walk-off parameter $\delta$ the properties of the walking solitons at either side of phase matching are no longer similar to each other at all values of the wavevector mismatch $\beta$, as they are for the nonwalking solitons that exist when $\delta=0$. Also, when $\delta=0$, all the solitons are self-similar at exact phase-matching, but this is no longer so for the families of walking solitons. Physically, such differences are a consequence of the fact that the wave-front curvatures induced by the Poynting vector walk-off modify the effective wave-vector mismatch experienced by the different portions of the fundamental and the second-harmonic beams.

Similarly, in the absence of Poynting vector walk-off, as far as the soliton families are concerned, an increase in the wave-vector mismatch $\beta$ can be partially compensated for at the expense of a corresponding increase of the total wave intensity $I$, as the solitons in both cases differ only
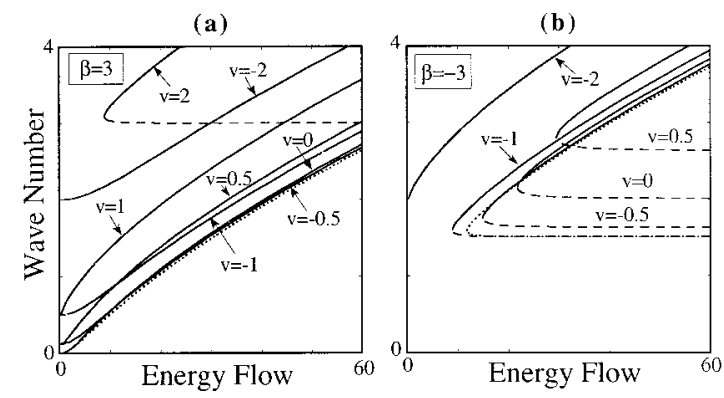

Fig. 1. Nonlinear wave-number shift versus energy flow for the families of walking solitons with different soliton velocities. (a) Solitons at positive wave-vector mismatch $(\beta=3)$, (b) solitons at negative wave-vector mismatch $(\beta=-3)$. In all cases $\delta$ $=1$. Dashed curves, unstable solutions; dotted curves, nonwalking solitons that exist in the absence of Poynting vector walk-off ( $\delta=0)$.
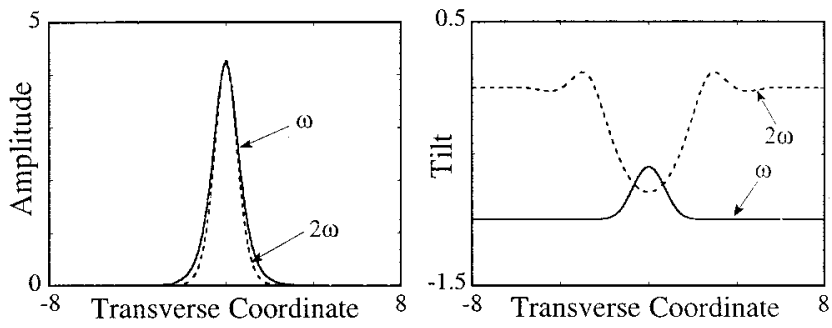

(a)
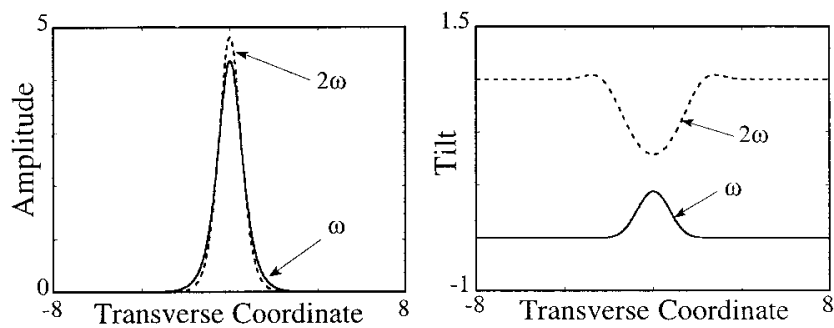

(b)
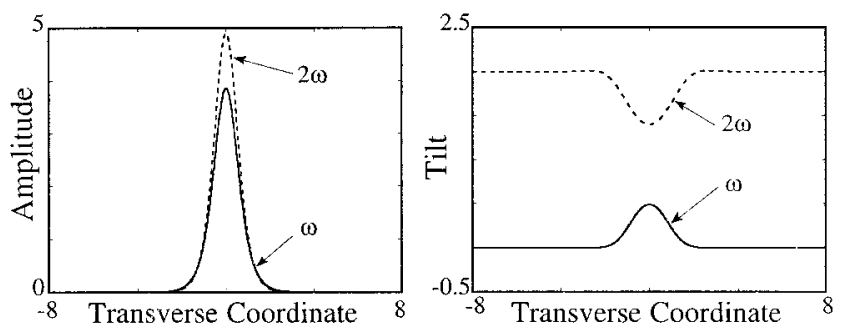

(c)
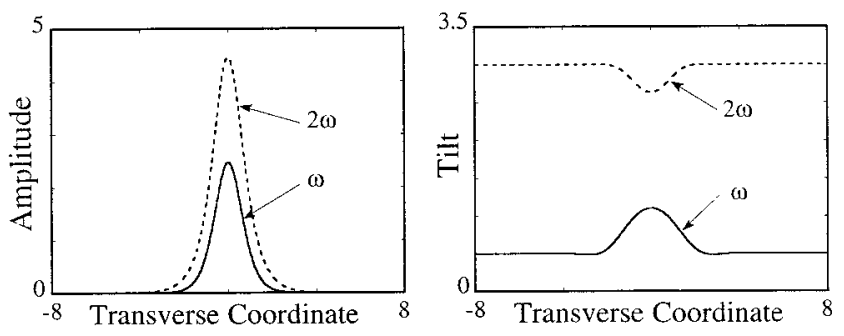

(d)

Fig. 2. Amplitude and local wave-front tilt, defined as the transverse derivative of the wave front, of solitons walking with different velocities at negative wave-vector mismatch, as a function of the transverse coordinate. Velocities: (a) $v=-1$, (b) $v$ $=-0.5$, (c) $v=0$, (d) $v=0.5$. In all cases $\beta=-3$ and $\delta=1$ and the nonlinear wave number shift is $\kappa_{1}=3$.

in amplitude and width. The scaling rules [transformations (23)] show that, for a given walk-off parameter $\delta$, such self-similarity no longer holds for the families of walking solitons. We shall have the occasion to use similarity rules (23) for the above purpose and for others in Section 5 .

\section{FAMILIES OF SOLITONS}

We obtained the families of stationary walking solitons by solving Eqs. (3)-(6) numerically, using a relaxation method with a band-diagonal matrix algorithm to deal with the two-point boundary value problem for the four unknown functions $U_{\nu}(\eta), f_{\nu}(\eta)$. Families of walking 

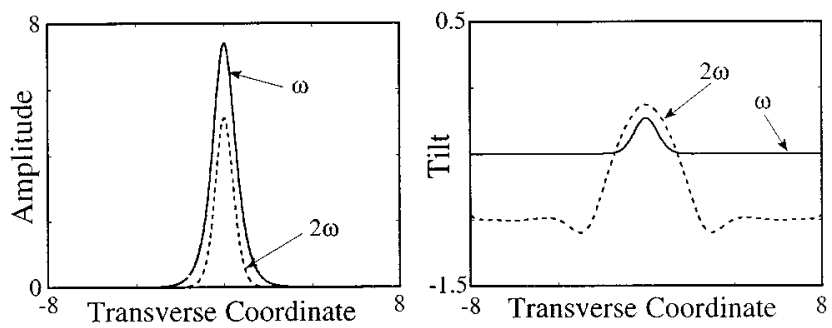

(a)
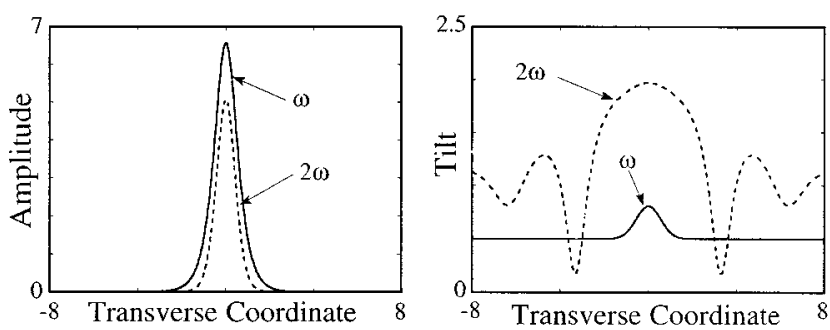

(b)

Fig. 3. Same as in Figs. 2(b) and 2(d) but for the walking solitons existing at positive wave-vector mismatch $(\beta=3)$. Velocities: (a) $v=-0.5$, (b) $v=0.5$. In both cases $\delta=1$.
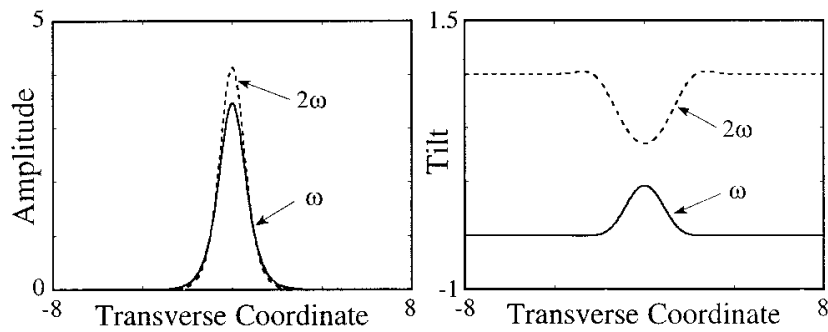

(a)
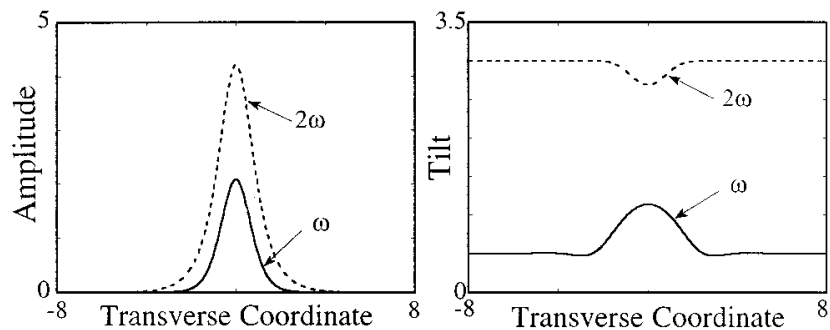

(b)

Fig. 4. Same as in Figs. 2(b) and 2(d) but for two walking solitons carrying the same energy flow. Conditions: $\beta=-3, I$ $=30$. Velocities: (a) $v=-0.5$, (b) $v=0.5$. In both cases $\delta$ $=1$.

solitons were found to exist with different values of the linear wave and material parameters $\alpha, \beta$, and $\delta$ at ranges of the wave intensities and soliton velocities.

For given values of $\alpha, \beta$, and $\delta$ the families of walking solitons depend on two independent parameters, namely, $\kappa_{1}$ and $v$, and a convenient way to represent them is with an energy-flow nonlinear wave-number diagram, i.e., $\kappa_{1}(I)$, with different values of $v$. Figure 1 shows such diagram at two different values of $\beta$, corresponding to both signs of the wave-vector mismatch, at a representative value of the walk-off parameter. We have also included the curves corresponding to the nonwalking soli-

tons that exist in the absence of walk-off. Walking solitons also exist at exact phase matching, and the corresponding figure is given in Ref. 20 .

The shape and related properties of the walking solitons depend strongly on the various parameters involved and in particular on their velocity. In Figs. 2 and 3 we have plotted the amplitude and the local wave-front tilt, defined as $\dot{f}_{\nu}(\eta)$, for several representative cases at $\delta$ $=1$. Figure 2 corresponds to a negative wave-vector mismatch and Fig. 3 to a positive wave-vector mismatch. In both cases the nonlinear wave-number shift $\kappa_{1}$ is kept constant, and the plots correspond to different soliton velocities. The differences in shape between solitons walking with different velocities are clearly visible. To interpret the results of these plots it is important to recall that for given values of $\delta$ and $\beta$, and a fixed value of $\kappa_{1}$, solitons walking with different velocities also carry a different total energy flow $I$. This fact can be observed directly from Fig. 1. The quantity that can be directly controlled experimentally is not the soliton parameter $\kappa_{1}$ but the energy flow $I$. Figure 4 shows the shapes of the walking solitons in the conditions of Figs. 2(b) and 2(d) but now for two walking solitons carrying the same energy flow.

The different behavior of the wave-front tilts in the soliton tails depending on whether condition (20) is verified is also visible in Figs. 2-4. When $\delta=1$ and $\beta$

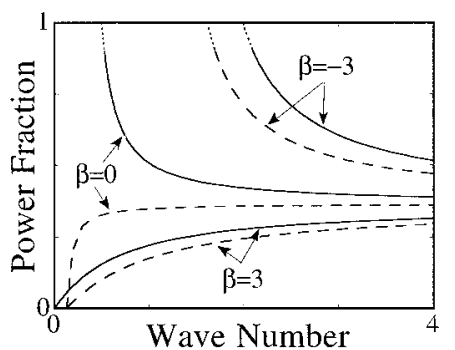

Fig. 5. Fraction of power carried by the second-harmonic beam (i.e., $I_{2} / I$ ) as a function of the nonlinear wave-number shift $\kappa_{1}$ at several wave-vector mismatches and soliton velocities. Solid curves, walking solitons with $v=0$; dashed curves, solitons walking with the velocity $v=-0.5$. The short portions of the curves near $I_{2} / I=1$ plotted as dotted curves stand for the corresponding unstable solutions. In all cases $\delta=1$.

(a)

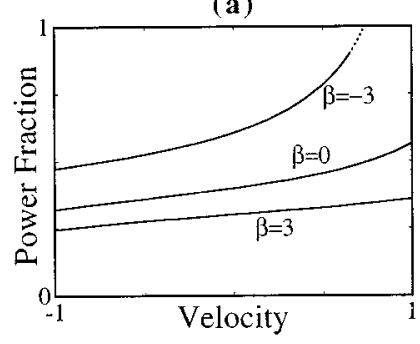

Fig. 6. Fraction of power carried by the second-harmonic beam as a function of the soliton velocity at several wave-vector mismatches. Conditions: in (a) the nonlinear wave-number shift is fixed to $\kappa_{1}=3$; in (b) the total energy flow is fixed to $I=40$. The short portion of the curve corresponding to $\beta=-3$ near $I_{2} / I=1$ plotted as a dotted curve stands for unstable solutions. In all cases $\delta=1$. 
$=-3$, expression (20) leads to $v>-2$. All cases shown in Fig. 2 verify this condition; hence $\dot{f}_{2}$ decays monotonically and tends to $2(\delta+v)$. In opposition, at $\beta=3$ condition (20) demands $v>1$; thus the walking solitons shown in Fig. 3 fall outside this range. The resulting complicated, oscillatory behavior of the local wave-front tilt of the second-harmonic beam can be clearly observed in Fig. 3.

The amplitudes and wave-front tilts of the two waves that form the walking solitons are related by a first integral of Eqs. (4) and (6). That is,

$$
\left[\dot{f}_{1}(\eta)-v\right] U_{1}^{2}(\eta)-\left[\alpha \dot{f}_{2}(\eta)+v+\delta\right] U_{2}^{2}(\eta)=0 .
$$

The two terms of Eq. (24) do not vanish separately, as they do for traveling-wave solutions, but rather they cancel each other. This result has interesting consequences. For example, writing general fields as $a_{\nu}=U_{\nu} \exp \left(i \phi_{\nu}\right)$, and calculating the local energy flux at each frequency, in the frame of reference moving with transverse velocity $v$, one readily finds

$$
\begin{aligned}
\frac{\partial U_{1}^{2}}{\partial \xi}= & -\frac{\partial}{\partial \eta}\left[\left(\frac{\partial \phi_{1}}{\partial \eta}-v\right) U_{1}^{2}\right] \\
& -2 U_{1}^{2} U_{2} \sin \left(\phi_{2}-2 \phi_{1}-\beta \xi\right), \\
\frac{\partial U_{2}^{2}}{\partial \xi}= & \frac{\partial}{\partial \eta}\left[\left(\alpha \frac{\partial \phi_{2}}{\partial \eta}+v+\delta\right) U_{2}^{2}\right] \\
& +2 U_{1}^{2} U_{2} \sin \left(\phi_{2}-2 \phi_{1}-\beta \xi\right) .
\end{aligned}
$$

In the case of stationary solitons one has $U_{\nu}=U_{\nu}(\eta)$, so that the local energy fluxes vanish. Thus so do the righthand sides of both Eqs. (25) and (26). The first terms on the right-hand sides of these expressions can be viewed as the local energy flux that is due to the transverse movement of the beams, whereas the second terms stand for nonlinear energy transfer between the fundamental and the second-harmonic waves. The point is that the two contributions to the local energy flux do not vanish separately, as they do for traveling-wave solutions with a flat wave front. Rather, they cancel each other. This can be interpreted to mean that, in contrast to nonwalking solitons or to traveling-wave solutions, in the case of general walking solitons the energy is continuously transferred between the interacting waves that form the soliton and at the same time is redistributed inside the beams because of the wave-front curvature so that the net energy flux vanishes.

When $\delta=0$ and $\alpha=-0.5$ both terms in Eq. (24) vanish separately, and then a first integral of Eqs. (3) and (5) gives the relation between the peak amplitudes of the two waves that form the solitons. ${ }^{26}$ That is,

$$
U_{1}^{2}(0)=\frac{2 \kappa_{1}+\beta}{2\left[U_{2}(0)-\kappa_{1}\right]} U_{2}^{2}(0) .
$$

The features of the shapes and wave fronts of the solitons have crucial implications for their actual experimental excitation. In particular, Fig. 1 shows that even in the presence of walk-off there are zero-velocity soliton solutions. However, the excitation of such solitons requires an input beam that exactly matches the stationary solu- tion, including its precise wave-front curvature. Anyway, solitons walking with quite a small velocity can be always excited with appropriate tilted inputs. This is so provided that the Poynting vector walk-off is reasonably small, and it is of course at the expense of losing a fraction of the input light in the form of radiation.

Similarly, Fig. 1(b) shows that at negative wave-vector mismatch walking solitons can exist at energy flows lower than the minimum threshold energy required for the existence of nonwalking solitons in the absence of walk-off. This situation is also due to the wave-front curvatures of the walking solitons. The actual excitation of the lowenergy solitons with nonspatially chirped beams produces a significant amount of radiation. We return to these points in Section 7.

To stress the important role played by the velocity in the family of walking solitons, we show in Figs. 5 and 6 how the total energy of the walking solitons is shared between the fundamental and the second-harmonic waves as a function of the nonlinear wave number shift and the soliton velocity. Notice that in Fig. 6(a) the parameter $\kappa_{1}$ is fixed, whereas Fig. 6(b) corresponds to a fixed total energy flow. Figure 7 shows the widths (full width at halfmaximum) of the two beams that form the soliton as a function of the soliton velocity. In Fig. 7(a) the parameter $\kappa_{1}$ is fixed, whereas Fig. 7(b) shows the widths of the beams that form the different walking soliton for a fixed energy flow $I$. The dotted curves in Figs. 5-7 stand for the unstable solutions that exist in the cases that we examine. Because unstable solutions occur near cutoff for the existence of a soliton, they have correspondingly large beam widths. Notice also that as one approaches the cutoff almost all the energy flow of the soliton is carried either by the fundamental beam or by the second harmonic.

To end this section, Fig. 8 shows the dependence of the threshold energy for the existence of solitons walking with the specific velocities $v=-\delta / 2$ and $v=-\delta / 4$ as a function of the walk-off parameter $\delta$ for various values of the wave vector mismatch $\beta$. Consistent with the scaling rules [transformations (23)], at exact phase matching the threshold energy for the existence of solitons with the velocity $v=-\delta / 4$ increases as $\delta$ to the power of 3 . Outside phase matching, such dependence has to be calculated numerically. At $\beta=0$ there is no cutoff for the existence of solitons walking with the velocity $v=-\delta / 2$, consistent with the curves of Fig. 1 . Similarly, at $\beta=3$ there is no threshold energy for the existence of solitons in the range of walk-off parameters and soliton velocities displayed in the plot of Fig. 8.

\section{STABILITY}

The stability of the families of walking solitons can be elucidated in various ways. A generic stability criterion for two-parameter families of solitons was reported by Buryak et al., ${ }^{11}$ using a linear stability analysis, that therefore formally holds for the families of walking solitons. Etrich and co-workers ${ }^{22}$ specifically addressed the stability criterion of walking solitons by using a similar approach and examined its application to the case of temporal solitons. They found the condition of marginal linear stability 

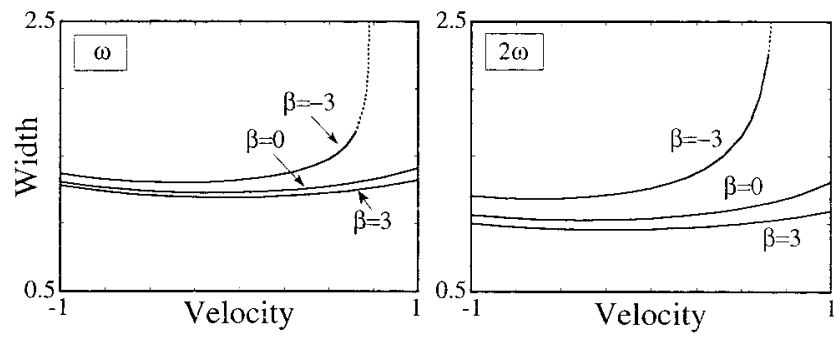

(a)
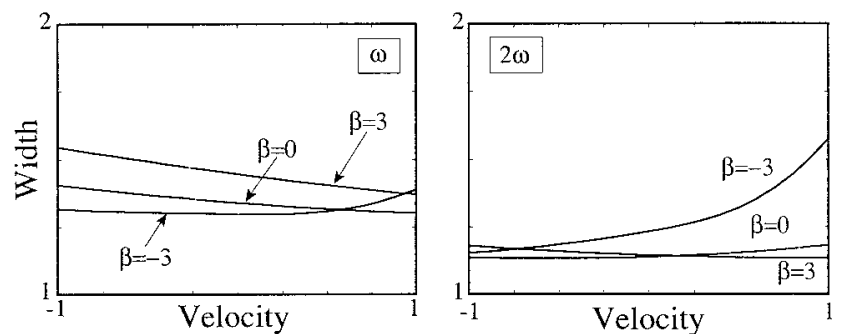

(b)

Fig. 7. Widths (FWHM) of the fundamental and the secondharmonic beams of representative walking solitons that exist at several wave-vector mismatches as a function of the soliton velocity. In (a) the nonlinear wave-number shift is fixed to $\kappa_{1}$ $=3$; in (b) the total energy flow is fixed to $I=40$. In all cases $\delta=1$.

$$
\frac{\partial I}{\partial \kappa_{1}} \frac{\partial J}{\partial v}-\frac{\partial I}{\partial v} \frac{\partial J}{\partial \kappa_{1}}=0
$$

Here we outline a geometrical interpretation of the stability criterion, and show in detail its implications for the families of spatial walking solitons. The geometrical interpretation of the stability criterion gives direct insight into the global stability of the solitons and also stresses the universality of the criterion for similar systems.

Recall that we refer to the stability of the solitons when they are propagated in the system with the same number of transverse dimensions where they have been found. In other words, modulational instabilities against higherdimensional perturbations are not considered. Otherwise, solitons in quadratic nonlinear media are known to be modulationally unstable in both $(1+1)$ and $(2+1)$ geometries if the corresponding perturbations are allowed to grow. ${ }^{27,29}$

Our starting point is variational expression (10). Because the families of stationary walking solitons realize the extrema of $H$ for given $I$ and $J$, one concludes that solutions that realize the global minimum of $H$ are stable. Solutions that realize a local minimum can be stable against small enough perturbations that keep the perturbed solution inside the local well of $H$. These solutions can be called metastable. Solutions that realize a local maximum of $H$ are always unstable. Therefore, to elucidate the stability of the families of walking solitons, one might examine the surface $H=H(I, J)$ and identify its lower and upper sheets. Solutions belonging to the lower sheet will be stable under propagation. Solutions belonging to upper sheets will be metastable or unstable, a condition that can be elucidated, e.g., by numerical experiments. In that case the curve of marginal stability, which separates stable from possibly unstable solutions, is the curve that separates the lower and upper sheets of the $H=H(I, J)$ surfaces.

In the case of smooth $H=H(I, J)$ surfaces determination of the marginal curve is elementary when we notice that over the curve the vector normal to the surface is contained on the horizontal plane. The vector normal to the two-parametric surface $\mathbf{r}=I\left(\kappa_{1}, v\right) \hat{x}+J\left(\kappa_{1}, v\right) \hat{y}$ $+H\left(\kappa_{1}, v\right) \hat{z}$ is given by the expression

$$
\mathbf{n}=\frac{\partial(J, H)}{\partial\left(\kappa_{1}, v\right)} \hat{x}+\frac{\partial(H, I)}{\partial\left(\kappa_{1}, v\right)} \hat{y}+\frac{\partial(I, J)}{\partial\left(\kappa_{1}, v\right)} \hat{z},
$$

where the symbol $\partial(F, G) / \partial(a, b)$ stands for a Jacobian matrix. The vector $\mathbf{n}$ is contained on the horizontal plane when the last term on the right-hand side of Eq. (29) vanishes, yielding Eq. (28). There is no need to assume that the surface $H=H(I, J)$ is smooth everywhere. It may exhibit sharp foldings at the marginal curve where lower and upper sheets join each other, as happens for nonwalking solitons. ${ }^{30}$ In that case condition (28) can be viewed as indicating a change of sign of the vertical component of $\mathbf{n}$, thus signalling the change of sheet. The surface $H=H(I, J)$ may still exhibit sheet crossings where a change of sheet is not necessarily accompanied by a change of sign of the vertical component of $\mathbf{n}$. Those cases ought to be treated separately, and global stability of the corresponding portions of the upper sheets ought to be carefully checked.

Criterion (28) is more involved than the so-called Vakhitov-Kolokolov criterion, ${ }^{31}$ given by

$$
\frac{\partial I}{\partial \kappa_{1}}=0,
$$

that holds for nonwalking solitons. ${ }^{30,32}$ Geometrically, we derive this criterion by noticing that the nonwalking solitons that exist in the absence of walk-off realize the minimum of $H$ for a given $I$, namely, $\delta_{F}\left[H+\kappa_{1} I\right]_{\text {sta }}=0$. Therefore in this case the surface $H=H(I, J)$ is reduced to the curve $H=H(I)$, and the condition of marginal stability is given by the point that separates the lower from the upper branches of the parametrically defined curve $\left\{I=I\left(\kappa_{1}\right), H=H\left(\kappa_{1}\right)\right\}$. Examination of the corresponding curves, calculated in Ref. 30 and shown here in

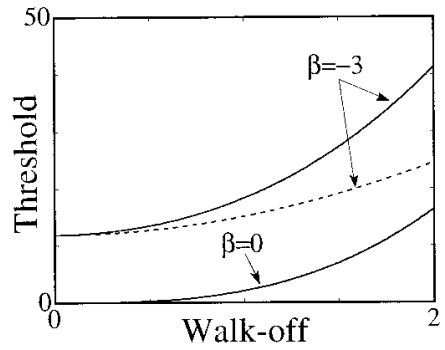

Fig. 8. Threshold (minimum) energy flow for the existence of solitons walking with a given velocity as a function of the walkoff parameter $\delta$ for various values of the wave-vector mismatch. Solid curves, threshold energy for solitons walking with a velocity $v=-\delta / 4$; dashed curve, threshold energy for solitons walking with a velocity $v=-\delta / 2$. The plot contains only one curve for $\beta=0$ and none for $\beta=3$, because in the missing cases there is no threshold energy for the existence of solitons in the range of walk-off parameters and soliton velocities displayed. 

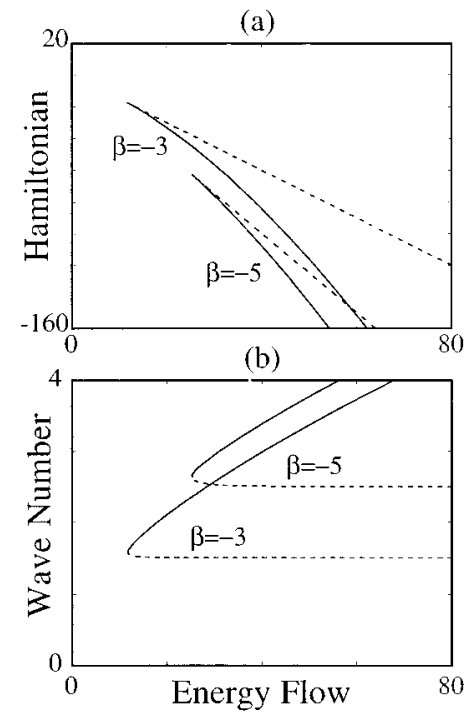

Fig. 9. Energy Hamiltonian curves and energy wave-number curves to determine geometrically the stability of the nonwalking solitons that exist in the absence of Poynting vector walk-off ( $\delta$ $=0)$. Solid curves, stable solitons; dashed curves, unstable solutions. (From Ref. 30).

(a)

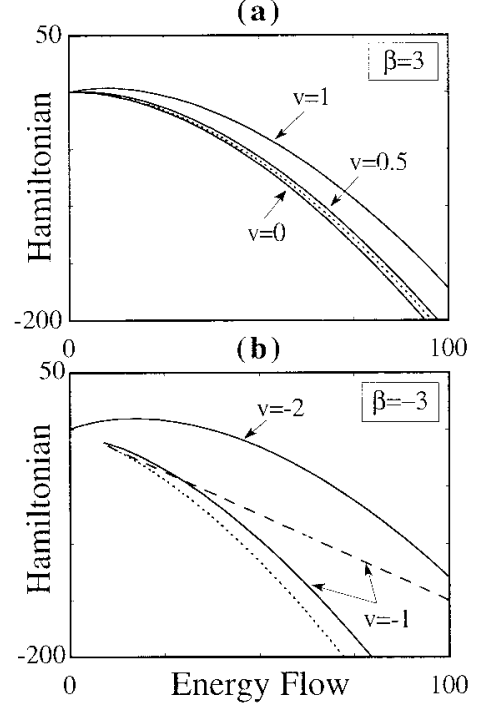

Fig. 10. Hamiltonian versus energy flow for some of the families of walking solitons. Notice that the curves correspond to fixed soliton velocities; therefore the momentum is not constant along the curves. For the sake of clarity, only the curves for a few velocities are shown. Solid curves, stable walking solitons; dashed curves, unstable solutions; dotted curves, nonwalking solitons that exist in the absence of Poynting vector walk-off $(\delta=0)$. A zoom of the curve corresponding to $\beta=-3, v=-1$ near the point of marginal stability is shown in Fig. 12(b) below.

Fig. 9, shows that lower and upper branches join each other at a sharp cusp and leads immediately to condition (30). The rigorous mathematical proof is given by Whitney's theorem about two-dimensional maps applied to this case. ${ }^{33}$

To identify the stable and the unstable walking solitons, one has to examine the geometrical features of the $H=H(I, J)$ surfaces, in particular evaluating the Jacobian Eq. (28), for the families of solutions. However, no- tice that only the families that exhibit multivalued surfaces ought to be studied in detail. Otherwise the existing solutions are always found to correspond to the lower sheet of the $H=H(I, J)$ surfaces, and all members of the family are found to be stable. The whole surfaces $H=H(I, J)$ are not easily visualized because of their asymmetry relative to the $I$ and $J$ axes. Figures 10 and 11 show the typical features of the projections in the $I-H$ and in the $I-J$ planes of the curves of the surfaces corresponding to some of the families of walking solitons that appear in Fig. 1. As predicted by Eq. (13), Fig. 11 shows that the zero-velocity solitons carry a nonvanishing momentum: They need the corresponding wave-front cur-

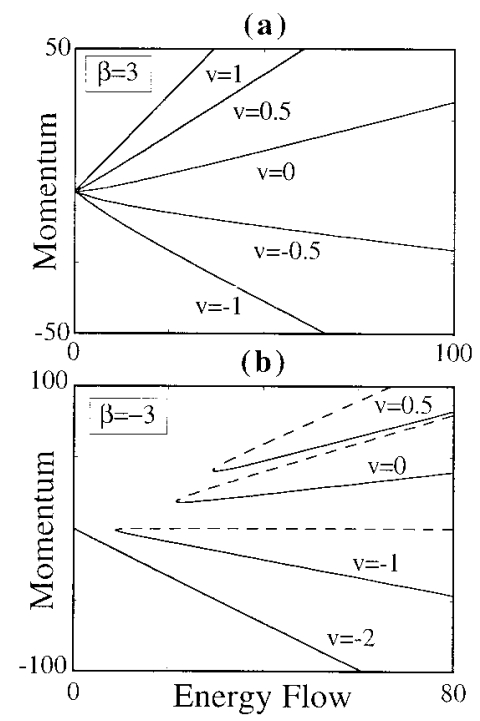

Fig. 11. Momentum versus energy flow for the families of walking solitons. Notice that the Hamiltonian is not constant along the curves. Solid curves, stable walking solitons; dashed curves, unstable solutions.

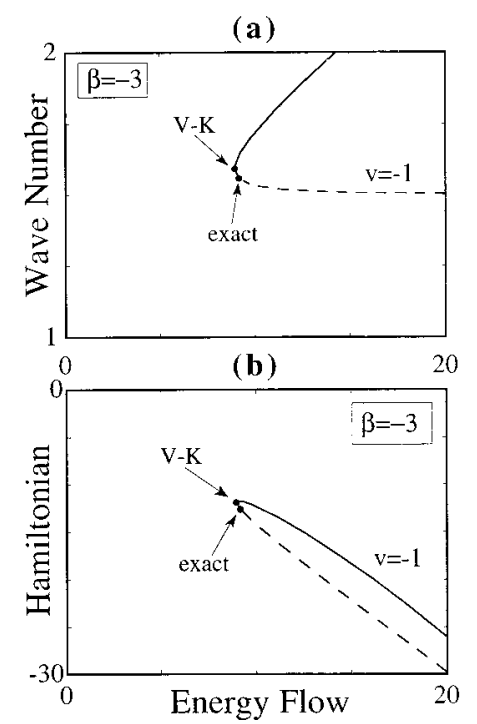

Fig. 12. Zooms of two representative curves of Figs. 1(b) and Fig. 10(b) that show the small differences that exist between the numerical values for the condition of marginal stability given by the exact criterion [Eq. (28)] and by the Vakhitov-Kolokolov (V-K) criterion [Eq. (30)]. The curves correspond to $\beta=-3$, $v=-1$. 


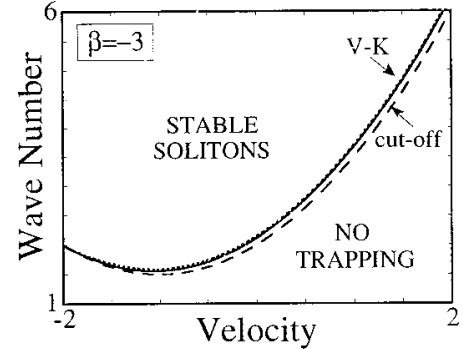

Fig. 13. Summary of the stability analysis for the family of solitons that exist at $\beta=-3, \delta=1$, with velocities in the range $[-2,2]$. Values of the nonlinear wave-number shift $\kappa_{1}$ are shown at the cutoff for the soliton existence (dashed line) and at the condition of marginal stability (solid curve). The dotted curve that almost coincides with the solid curve corresponds to the condition of marginal stability given by criterion (30). Above the solid curve, all solutions are stable. The existing unstable solutions occur in the narrow band that appears between the cutoff and the marginal stability lines.

vature to cancel the linear walk-off otherwise experienced by the second-harmonic beam.

As indicated by Fig. 11, we found that there are regions where the surface $H=H(I, J)$ has one single sheet and regions where it has two sheets. This does not exclude the possibility that additional upper branches exist in narrow regions near the cutoff condition for the soliton existence, similar to those appearing in the case of nonwalking solitons in type II geometries. ${ }^{11}$ However, walking solitons near their cutoff are correspondingly wide, yielding diffraction lengths in excess of $1 \mathrm{~m}$. Therefore a systematic scan of the $H=H(I, J)$ surfaces near the cutoff edge for the soliton existence falls outside the scope of this paper.

The outcome of the stability criterion for the families of walking solitons was already included in Fig. 1. It turns out that, for all the families of walking solitons considered, the numerical difference between the conditions of marginal stability given by Eqs. (28) and (30) is extremely small. A representative case is displayed in Fig. 12. The plots are zooms of the region of the corresponding curves near the condition of marginal stability and show that the numerical values given by Eqs. (28) and (30) almost coincide. Accordingly, the corresponding difference has no experimental relevance in the cases studied here. This fact is also supported by the result of previous comprehensive series of numerical simulations for selected members of the families of walking solitons, ${ }^{20}$ which showed that criterion (30) approximately holds for the families of spatial walking solitons that we are addressing. Notice that this conclusion does not necessarily hold for mathematically possible, large values of the walk-off parameter $\delta$ and soliton velocity $v$. However, one has to keep in mind that $\chi^{(2)}$ crystals are birefringent, so at such large values of $\delta$ or $v$ the reduced, scalar, and paraxial governing equations (1) might not hold. The situation may be different in the case of temporal solitons, ${ }^{22}$ because of the existing large group-velocity mismatches between the fundamental and the second-harmonic pulses and because of the additional degree of freedom offered by the values of the group-velocity dispersion of the waves at both frequencies.
To monitor the evolution of the stable and the unstable solutions we performed the corresponding series of numerical experiments by propagating a variety of stationary solutions. The numerics always confirmed the above expectations. The unstable solutions either spread or reshaped and excited stable oscillating walking solitons that converge to the lower sheet of the $H=H(I, J)$ surface.

Figure 13 summarizes the stability analysis for all the families of solitons that exist at $\beta=-3$ with $\delta=1$, walking with velocities in the range $[-2,2]$. Analogous results are obtained at other values of the wave-vector mismatch. The plot has to be interpreted as follows: Soliton solutions exist for nonlinear wave-number shifts above the cutoff line, which here is given by expression (21). All the solutions that exist above the solid curve are stable. The narrow band that appears between the stability and the cutoff lines corresponds to the unstable solutions. The condition of marginal stability given by criterion (30) is shown by a dotted curve, even though one has to examine the plot carefully to observe it because it almost coincides with the exact stability threshold.

The main conclusion to be drawn is that most of the members of the families of spatial walking solitons are stable on propagation.

The existence and stability conditions of stationary walking solitons are summarized in Fig. 14. The curves correspond to wave-vector mismatches $\beta= \pm 3$ and to the walk-off $\delta=1$, but analogous results are obtained at other values of these parameters. The figure emphasizes that above the energy threshold for soliton formation there is always a stable solution. When interpreting Fig. 14 from an experimental viewpoint it is worth recalling that low-energy solitons have a correspondingly large width; thus for a given beam width there is always an energy threshold for soliton formation. Also, the threshold energy shown in the plots corresponds to the families of
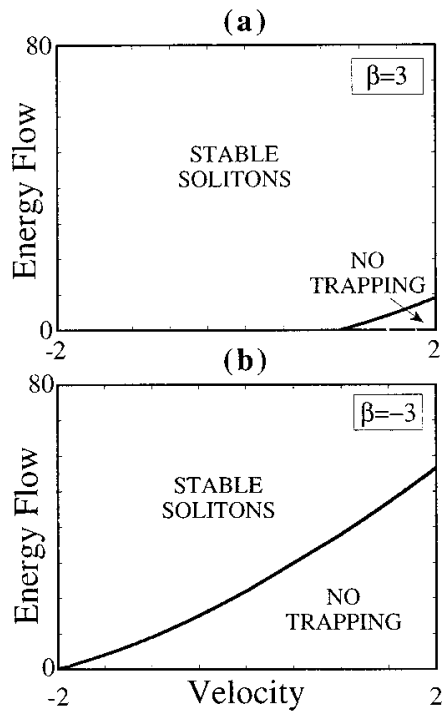

Fig. 14. Threshold energy flow for stationary solitons to exist as a function of the soliton velocity, at two values of the wave-vector mismatch. Above the existence curve there is always a stable walking soliton solution. 
(a)
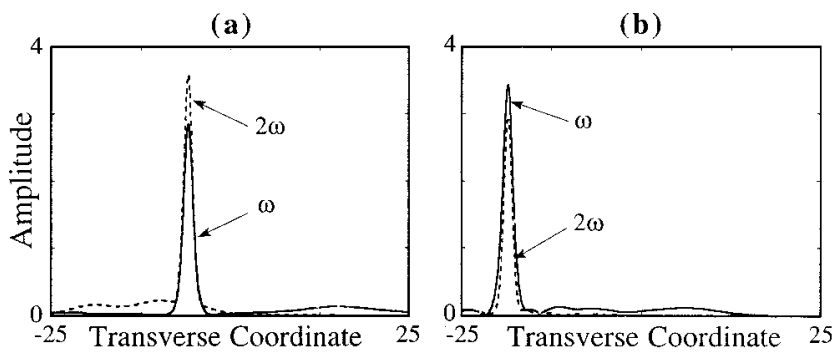

Fig. 15. Typical excitation of a slow and a fast walking soliton. Amplitudes of the fundamental and the second harmonic beams at $\xi=10$ of two selected walking solitons that have been numerically excited with tilted input beams and with different input conditions. Tilts: (a) $\mu=0$, (b) $\mu=-1.5$. In both cases $A=3, B=3, \beta=-3$, and $\delta=1$.

exact stationary solutions whose features do not coincide with the input light that is used in practice to excite the solitons.

\section{EXCITATION}

The excitation of walking solitons with different input beams is governed to a large extent by Eq. (13). For our present purposes it is better to write Eq. (13) as

$$
v=\frac{J}{I}-\delta \frac{I_{2}}{I}-(2 \alpha+1) \frac{J_{2}}{I} .
$$

This expression has to be used with caution because it holds for the families of stationary walking solitons but not for the input light conditions. The difference is that, unless the input conditions exactly match the shape of the walking soliton solutions, the beam dynamics and reshaping toward the formation of a walking soliton always produce some radiation that takes energy and momentum away. Therefore in such a case the momentum $J$ of the soliton that eventually appears is not equal to the input value. Also, $I_{2}$ and $J_{2}$ are not even conserved quantities of the beam evolution, so their values do change dynamically during propagation. In particular, the dynamic evolution of $I_{2}$ depends significantly on the material parameters, including the value of $\delta$, and on excitation conditions. ${ }^{16}$ However, when the radiation produced is small, as happens with many typical input conditions, Eq. (31) provides a direct estimation of the velocity of the walking soliton that eventually gets excited.

The last term on the right-hand-side of Eq. (31) is very small. The first term of such an equation accounts for the effect of any tilts or for more-complicated transverse phase modulations of the input beams. ${ }^{34}$ Finally, the second term stands for the mutual dragging of the fundamental and the second-harmonic beams. In particular, that term shows that zero-momentum inputs, i.e., without any wave-front tilt, excite walking solitons with a velocity $v \sim-\delta I_{2} / I$. To estimate the soliton velocity one can use the value of the ratio $I_{2} / I$ corresponding to the family of stationary walking solitons. This ratio happens to depend strongly on the linear wave-vector mismatch and also on the total energy flow. ${ }^{26}$ By and large, at positive $\beta$ the solitons have small $I_{2} / I$; thus they walk slowly. At phase matching and at negative $\beta$ the solitons have larger $I_{2} / I$; therefore they walk faster. With high energy flows the absolute value of the effective wave-vector mismatch is smaller than the linear value given by $\beta,{ }^{26}$ regardless of its sign; thus the ratio $I_{2} / I$ and the soliton velocity $v$ behave accordingly. For example, at positive $\beta$ higher-energy solitons walk faster than lower-energy solitons.

The formation of walking solitons under a variety of excitation conditions was shown in Refs. 8 and 16 for several typical cases; hence there is no need to discuss those most common situations here. Rather, we show the excitation of walking solitons in a few selected cases that we have chosen to stress that all the families of walking solitons for different $\delta$ and $v$ are physically relevant. Naturally, we provide the examples that we consider judicious values of $\delta$ and $v$ for which the paraxial and scalar approximations required to derive Eqs. (1) hold.

To excite a walking soliton with a large velocity one can use tilted input beams, with the form $a_{1}(\xi=0, s)$ $=A U(s) \exp (i \mu s)$ and $a_{2}=B V(s) \exp (i 2 \mu s)$, where $U$ and $V$ are the transverse profiles of the input beams, $A$ and $B$ are their amplitudes, and $\mu$ is the wave-front tilt. To show a representative case we selected $\beta=-3$ and $\delta=1$, and for this case we set $U(s)=V(s)=\operatorname{sech}^{2}(s)$. Figures 15 and 16 show the excited walking solitons for different input conditions. Figure 15(a) corresponds to an input with $\mu=0$, and Fig. 15 (b) to an input with $\mu$ $=-1.5$. The salient point of the plots is that in Fig. 15(a) the second-harmonic beam is higher than the fundamental, as happens for the solitons that exist in the absence of Poynting vector walk-off at this value of $\beta,{ }^{26}$ but in the case of a soliton walking with a velocity $v \approx-1.9$ that appears in Fig. 15(b) the fundamental beam carries the largest energy. This result is consistent with the properties of the families of walking solitons as a function of the velocity as illustrated in Figs. 2, 5, and 6. Physically, this is so because the wave-front curvatures of the walking solitons modify the effective wave-vector mismatch across the beams from the nominal value given by $\beta$.

Some curves shown in Fig. 1 correspond to solitons that walk against the Poynting vector walk-off. Figure 16(a) shows one such soliton that that we have excited with $A$ $=4$ and $B=4$ and forced to walk against the Poynting vector walk-off by setting $\mu=1$. Finally, Fig. 16(b) shows the case of a low-energy soliton walking with a velocity $v \approx-1.7$ that is formed at $\beta=-3$ with $A=1, B$ (a)

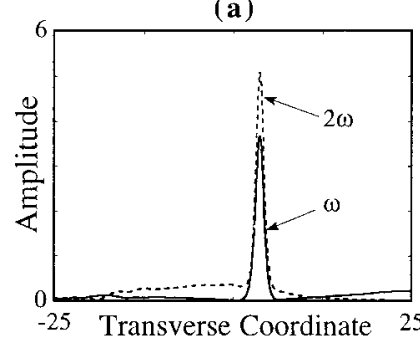

(b)

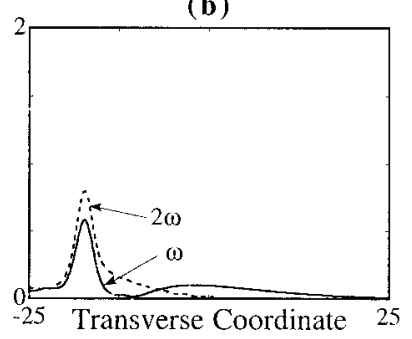

Fig. 16. Excitation of two rare walking solitons. (a) Soliton that walks against the Poynting vector walk-off. (b) Walking soliton that carries an energy much smaller than the minimum required for formation of a nonwalking soliton. Conditions: (a) $A=4, B=4, \mu=1$; (b) $A=1, B=1, \mu=-1$. Conditions and features are as in Fig. 15. 
$=1$, and $\mu=-1$. It is worth recalling that, at $\beta$ $=-3$, nonwalking solitons in the absence of Poynting vector walk-off do not exist at such low energies, but walking solitons do.

To end this section and the paper, we note that an analogous analysis can be used to investigate the properties of walking solitons of type II phase-matching geometries. The corresponding evolution equations are given in Appendix A. Because type II geometries involve three different waves, namely, two fundamental beams with different polarizations and one second-harmonic beam, the walking solitons constitute a three-parameter family. Physically, such parameters are related to the total energy flow, to the energy difference between two of the involved beams (e.g., the two fundamental beams), and to the soliton velocity. The extra degree of freedom relative to type I geometries, namely, the energy difference between two of the beams, has important implications. ${ }^{11}$ For example, it can be used to control the velocity of the walking solitons. In particular, the analogous Eq. (31) for type II geometries can be written as

$$
v=\frac{J}{I}-\delta_{2} \frac{I_{2}}{I}-\delta_{3} \frac{I_{3}}{I}-\left(\alpha_{2}+1\right) \frac{J_{2}}{I}-\left(2 \alpha_{3}+1\right) \frac{J_{3}}{I} .
$$

Changing the polarization of the light that is input at the fundamental frequency modifies the ratios $I_{2} / I$ and $I_{3} / I$; hence it changes the velocity of the walking soliton that is excited. Particular cases of this possibility were observed experimentally by Torruellas et al. ${ }^{18}$ and studied numerically by Leo and co-workers. ${ }^{19}$

\section{CONCLUDING REMARKS}

To summarize, we have investigated in detail the properties of the families of spatial walking solitons that exist in quadratic nonlinear media under conditions for type I second-harmonic generation in the presence of Poynting vector walk-off. The families of walking solitons constitute a two-parameter family, and they exist for wide ranges of physically relevant soliton energies and soliton velocities. The walking solitons have curved wave fronts, with nontrivial wave-front curvatures. We studied the shape and various experimentally relevant features of the solitons, their stability on propagation and their excitation, for different values of the linear wave-vector mismatch between the fundamental and the secondharmonic waves. We showed that most of the solitons are stable under propagation and that above the threshold energy flow for the existence of solitons there is always a stable walking soliton. The approach presented can be applied to other analogous but different physical settings in which similar multiple-wave walking solitons exist.

Note added in revision: Since our paper was submitted for publication several important papers about the topic that we address have been published. In particular, useful approximate analytical expressions of the families of walking solitons in type I geometries have been obtained, ${ }^{35}$ exact families of walking solitons in type II geometries and their stability have been elucidated, ${ }^{36}$ and potential applications of the walking solitons have been proposed and studied by Capobianco et al. ${ }^{37}$ and by Leo and co-workers. ${ }^{38}$

\section{APPENDIX A: TYPE II PHASE-MATCHING GEOMETRIES}

In this paper we focused on type I phase-matching geometries, as they are simpler and capture the basic features of walking solitons. However type II phase-matching geometries are richer and are important from the experimental viewpoint. The normalized evolution equations for the slowly varying field envelopes in type II phase matching geometries for second-harmonic generation can be written as

$$
\begin{array}{r}
i \frac{\partial a_{1}}{\partial \xi}-\frac{\alpha_{1}}{2} \frac{\partial^{2} a_{1}}{\partial s^{2}}+a_{3} a_{2} * \exp (-i \beta \xi)=0 \\
i \frac{\partial a_{2}}{\partial \xi}-\frac{\alpha_{2}}{2} \frac{\partial^{2} a_{2}}{\partial s^{2}}-i \delta_{2} \frac{\partial a_{2}}{\partial s}+a_{3} a_{1} * \exp (-i \beta \xi)=0 \\
i \frac{\partial a_{3}}{\partial \xi}-\frac{\alpha_{3}}{2} \frac{\partial^{2} a_{3}}{\partial s^{2}}-i \delta_{3} \frac{\partial a_{3}}{\partial s}+a_{1} a_{2} \exp (i \beta \xi)=0
\end{array}
$$

where $a_{1}, a_{2}$, and $a_{3}$ are the normalized envelopes of the ordinary polarized fundamental beam, the extraordinary polarized fundamental beam, and the second-harmonic beam, respectively. In the case of spatial solitons $\alpha_{1}$ $=-1, \alpha_{2} \simeq-1$, and $\alpha_{3} \simeq-0.5$. We obtain the equations for type I geometries from Eqs. (A1) by setting $a_{2}$ $=a_{1}=a_{\omega}, \alpha_{1}=\alpha_{2}=r, \alpha_{3}=\alpha, \delta_{2}=0, \delta_{3}=\delta$, and $a_{3}=a_{2 \omega}$. Equations (A1) have several conserved quantities, including the corresponding Hamiltonian. To obtain Eq. (32) we used the energy flow given by the Manley-Rowe relation

$$
I=I_{1}+I_{2}+I_{3}=\int\left(1 / 2\left|a_{1}\right|^{2}+1 / 2\left|a_{2}\right|^{2}+\left|a_{3}\right|^{2}\right) \mathrm{d} s
$$

and the transverse beam momentum

$$
\begin{aligned}
J= & J_{1}+J_{2}+J_{3}=\frac{1}{4 i} \int\left[\left(a_{1} * \frac{\partial a_{1}}{\partial s}-a_{1} \frac{\partial a_{1} *}{\partial s}\right)\right. \\
& +\left(a_{2} * \frac{\partial a_{2}}{\partial s}-a_{2} \frac{\partial a_{2}^{*}}{\partial s}\right) \\
& \left.+\left(a_{3} * \frac{\partial a_{3}}{\partial s}-a_{3} \frac{\partial a_{3}^{*}}{\partial s}\right)\right] \mathrm{d} s .
\end{aligned}
$$

The families of solitons in Type II geometries in the absence of Poynting vector walk-off were investigated in Refs. 11 and 39. Those are two-parameter families, whereas walking solitons constitute a three-parameter family. Physically such parameters are related to the total energy flow, the unbalancing energy, and the soliton velocity. The extra degree of freedom relative to type I geometries, namely, the unbalancing energy, has important implications. 


\section{ACKNOWLEDGMENTS}

This research has been supported by the Spanish Government under grant PB95-0768. We gratefully acknowledge support from the European Union through the $\mathrm{Hu}-$ man Capital and Mobility Programme. The numerical work was carried out at the Centre de Computacio i Comunicacions de Catalunya. The research of $\mathrm{N}$. N. Akhmediev is supported by the Australian Photonics Cooperative Research Center.

*Permanent address, Department of Theoretical Physics, Institute of Atomic Physics, Bucharest, Romania.

\section{REFERENCES}

1. Yu. N. Karamzin and A. P. Sukhorukov, Sov. Phys. JETP 41, 414 (1976).

2. G. I. Stegeman, D. J. Hagan, and L. Torner, Opt. Quantum Electron. 28, 1691 (1996).

3. R. Schiek, J. Opt. Soc. Am. B 10, 1848 (1993).

4. M. J. Werner and P. D. Drummond, J. Opt. Soc. Am. B 10, 2390 (1993).

5. K. Hayata and M. Koshiba, Phys. Rev. Lett. 71, 3275 (1993).

6. C. R. Menyuk, R. Schiek, and L. Torner, J. Opt. Soc. Am. B 11, 2434 (1994).

7. M. A. Karpierz and M. Sypek, Opt. Commun. 110, 75 (1994).

8. L. Torner, C. R. Menyuk, and G. I. Stegeman, Opt. Lett. 19, 1615 (1994); J. Opt. Soc. Am. B 12, 889 (1995); L. Torner and E. M. Wright, J. Opt. Soc. Am. B 13, 864 (1996).

9. A. V. Buryak and Y. S. Kivshar, Opt. Lett. 19, 1612 (1994); Phys. Lett. A 197, 407 (1995); A. V. Buryak, Y. S. Kivshar, and V. V. Steblina, Phys. Rev. A 52, 1670 (1995).

10. B. A. Malomed, D. Anderson, and M. Lysak, Opt. Commun. 126, 251 (1996).

11. A. V. Buryak, Y. S. Kivshar, and S. Trillo, Phys. Rev. Lett. 77, 5210 (1996); A. V. Buryak and Y. S. Kivshar, Phys. Rev. Lett. 78, 3286 (1997).

12. S. Trillo, Opt. Lett. 21, 1111 (1996).

13. C. Conti, S. Trillo, and G. Assanto, Phys. Rev. Lett. 78, 2341 (1997).

14. W. E. Torruellas, Z. Wang, D. J. Hagan, E. W. Van Stryland, G. I. Stegeman, L. Torner, and C. R. Menyuk, Phys. Rev. Lett. 74, 5036 (1995)

15. R. Schiek, Y. Baek, and G. I. Stegeman, Phys. Rev. E 53, 1138 (1996).
16. L. Torner, W. E. Torruellas, G. I. Stegeman, and C. R. Menyuk, Opt. Lett. 20, 1952 (1995).

17. W. E. Torruellas, Z. Wang, L. Torner, and G. I. Stegeman, Opt. Lett. 20, 1949 (1995).

18. W. E. Torruellas, G. Assanto, B. L. Lawrence, R. A. Fuerts, and G. I. Stegeman, Appl. Phys. Lett. 68, 1449 (1996).

19. G. Leo, G. Assanto, and W. E. Torruellas, Opt. Commun. 134, 223 (1996).

20. L. Torner, D. Mazilu, and D. Mihalache, Phys. Rev. Lett. 77, 2455 (1996).

21. D. Mihalache, D. Mazilu, L.-C. Crasovan, and L. Torner, Opt. Commun. 137, 113 (1997).

22. C. Etrich, U. Peschel, F. Lederer, and B. A. Malomed, Phys. Rev. E 55, 6155 (1997).

23. M. M. Fejer, G. A. Magel, D. H. Jundt, and R. L. Byer, IEEE J. Quantum Electron. 28, 2631 (1992).

24. E. A. Kuznetsov, A. M. Rubenchik, and V. E. Zakharov, Phys. Rep. 142, 103 (1986).

25. N. Akhmediev and M. Karlsson, Phys. Rev. A 51, 2602 (1995).

26. L. Torner, Opt. Commun. 114, 136 (1995).

27. A. A. Kanashov and A. M. Rubenchik, Physica D 4, 122 (1981).

28. A. De Rossi, S. Trillo, A. V. Buryak, and Y. S. Kivshar, Opt. Lett. 22, 868 (1997).

29. R. A. Fuerst, D.-M. Baboiu, B. Lawrence, W. E. Torruellas, G. I. Stegeman, S. Trillo, and S. Wabnitz, Phys. Rev. Lett. 78, 2756 (1997).

30. L. Torner, D. Mihalache, D. Mazilu, and N. N. Akhmediev, Opt. Lett. 20, 2183 (1995).

31. M. G. Vakhitov and A. A. Kolokolov, Sov. Radiophys. Quantum Electron. 16, 783 (1973); A. A. Kolokolov, Lett. Nuovo Cimento 8, 197 (1973).

32. D. E. Pelinovsky, A. V. Buryak, and Y. S. Kivshar, Phys. Rev. Lett. 75, 591 (1995).

33. H. Whitney, Ann. Math. 62, 374 (1954). Similar applications of this theorem can be found in books about catastrophe theory. See also F. V. Kusmartsev, Phys. Rep. 183, 1 (1989).

34. X. D. Cao, D. D. Meyerhofer, and G. P. Agrawal, J. Opt. Soc. Am. B 11, 2224 (1994).

35. A. D. Capobianco, B. Costantini, C. De Angelis, A. LauretiPalma, and G. F. Nalesso, J. Opt. Soc. Am. B 14, 2602 (1997).

36. D. Mihalache, D. Mazilu, L.-C. Crasovan, and L. Torner, Phys. Rev. E 56, 6294 (1997)

37. A. D. Capobianco, B. Costantini, C. De Angelis, A. LauretiPalma, and G. F. Nalesso, IEEE Photonics Technol. Lett. 9, 602 (1997).

38. G. Leo, G. Assanto, and W. E. Torruellas, J. Opt. Soc. Am. B 14, 3134 (1997).

39. U. Peschel, C. Etrich, F. Lederer, and B. A. Malomed, Phys. Rev. E 55, 7704 (1997). 